www.jmscr.igmpublication.org

Impact Factor 5.244

Index Copernicus Value: 83.27

ISSN (e)-2347-176x ISSN (p) 2455-0450

crossref DOI: _https://dx.doi.org/10.18535/jmscr/v4i10.50

Journal Of Medical Science And Clinical Research

\title{
Exchange Transfusion and Predictors of Outcome in Severe Falciparum Malaria
}

Authors

\section{AE Mathew, AJ Mathew, D Tariang, S Longtrai, GM Varghese}

\section{Introduction}

With an estimated 300-350 million cases and 1-3 million deaths, annually, malaria continues to be a major health concern especially in Sub-Saharan Africa and South-East Asia. ${ }^{1}$ In India malaria has staged a comeback after the early 1960s when an all-time low of malaria cases $(49,151$ in 1963) seemed to suggest the possibility of an impending eradication. During the last few years India has recorded about 2 million confirmed malarial cases and 1,000 deaths annually. These figures reveal just the tip of the iceberg as the WHO estimates about 15 million cases and 20,000 deaths annually. ${ }^{2}$ Severe malaria occurs when the effective treatment is delayed, either because of poor access to health care and delay in diagnosis or because the drugs given are ineffective in rapidly clearing the parasite. ${ }^{3}$ Non-immune individuals are particularly susceptible to severe falciparum malaria. Multiple organ dysfunction occurs once the disease takes a severe course, causing mortality as high as $50 \%$. There is little pharmaceutical interest in development of new drugs due to low monetary returns on investment ${ }^{4}$ and there have been no major advances since the extraction of artemesinin and its derivatives in the early 1970s. With the recent emergence of geographical areas of artemesinin resistance, ${ }^{5}$ research and development of new drugs as well as revisiting available treatment modalities is a pressing need.

Exchange transfusion has been recommended as an adjunct to antimalarial treatment of severe falciparum malaria. ${ }^{6}$ Exchange transfusion has been shown to rapidly reduce the systemic parasitic load, replacing it with unparasitized cells and is hypothesised to have beneficial effects on the microvasculature. ${ }^{7} \quad$ Following exchange transfusion, there is a reduction of immature, circulating ring forms of $P$. falciparum. These are the precursors of the mature organisms which reduce the membrane deformability of RBCs leading to sequestraton and obstruction of the microvasculature. ${ }^{8}$ The diseased RBCs containing the mature parasites are replaced by normally deformable RBCs. ${ }^{9}$ Whole blood exchange is postulated to reduce the circulating inflammatory cytokines like tumor necrosis factor(TNF)- $\alpha$ which activate adhesion molecules that increase binding, adhesion and sequestration of parasitized RBCs to the endothelium of the microvasculature though this does not always correlate with the clinical picture. ${ }^{10}$ A large body of un-randomised retrospective studies and case reports suggest a beneficial role for exchange transfusion in severe malaria. ${ }^{11,12,13}$ However, no randomised controlled trials have been performed and there are conflicting case reports and expert opinions on the 
subject $^{14} \mathrm{~A}$ meta-analysis of 8 studies comparing patients with severe malaria who did and did not receive exchange transfusion in adjunct to antimalarials showed no survival benefit for exchange transfusion. ${ }^{15}$ However, the patients in the exchange transfusion group were more ill, as per the WHO criteria for severe malaria and had higher parasitemia. Due to the lack of clear evidence and consensus on indications, benefits and dangers involved, or on practical details such as the volume of blood that should be exchanged no recommendations have been made for its use in the latest WHO guidelines for treatment of malaria. ${ }^{16}$

We undertook this retrospective study at the Dr. H. Gordon Roberts' Hospital, a 365 bedded secondary care hospital situated in Shillong, Meghalaya to evaluate the role of exchange transfusion in severe falciparum malaria and to identify predictors of adverse outcome. Meghalaya is a malaria-endemic zone with documented widespread chloroquine resistance ${ }^{17}$. All patients who are diagnosed with falciparum malaria receive an initial dose of $120 \mathrm{mg}$ artesunate intravenously. Following this they receive $60 \mathrm{mg}$ of artesunate twice daily for 4 days along with doxycycline $100 \mathrm{mg}$ orally twice a day. In addition children with severe malaria received intravenous quinine at an initial dose of 10 $\mathrm{mg} / \mathrm{kg} / \mathrm{dose}$ every 8 hours. Primaquine is also given to patients with mixed malaria with $P$. vivax infection. Exchange transfusion is a common adjunct to treatment for severe, complicated falciparum malaria in this hospital.

\section{Methodology}

All patients diagnosed to have smear-positive falciparum malaria or mixed infection with both Plasmodium falciparum and Plasmodium vivax who were admitted in the hospital from March 2009 to January 2010 were included in the study. Patients were classified as having severe falciparum malaria if they fulfilled one or more of the following criteria; ${ }^{18}$ 1) cerebral malaria 2) severe anaemia (Hematocrit $<15 \%$ or $\mathrm{Hb} \%<5$ $\mathrm{g} / \mathrm{dl}$ ) 3) jaundice with serum bilirubin $>3.0 \mathrm{mg} / \mathrm{dl}$ 4) renal failure with serum creatinine $>3.0 \mathrm{mg} / \mathrm{dl}$ 5) non cardiogenic pulmonary oedema 6) hypoglycaemia (blood glucose < $40 \mathrm{mg} \%$ ) 7) hypotension (systolic BP $<90 \mathrm{~mm} \mathrm{Hg}$ ) 8) bleeding (platelet count $<100,000 /$ cumm or deranged bleeding parameters) 9) metabolic acidosis $(\mathrm{pH}<7.25$ or $\mathrm{HCO} 3<15 \mathrm{mmol} / \mathrm{L}) 10$ ) hyperparasitemia (parasite index $>5 \%$ ). Data pertaining to age, sex, clinical features, blood pressure, laboratory parameters like haemoglobin, platelets, serum electrolytes, creatinine, liver function tests, urine output, blood transfusion, volume of exchange transfusion, anti-malarials used, use of inotropes, days of hospital stay and final outcome were obtained from the patient records. Statistical analysis was done using SPSS software. The association of clinical and laboratory parameters with the outcome were determined using Chi-square test. Logistic regression analysis was done to determine the predictors of mortality. The statistical significance was determined at $5 \%$ level.

\section{Results}

There were 219 patients (138 male and 81 female with a mean age of 20.96 years) treated for falciparum malaria or mixed infection. Of these54received exchange transfusion and $165 \mathrm{did}$ not. The average duration of fever before admission was $4.6 \pm 2.5$ days and average hospital stay was $6.08 \pm 3.6$ days. There were 10 patients who did not satisfy the criteria for severe malaria who had received exchange transfusion of which 2 were children. All had a haemoglobin less than 10 and 4 had a haemoglobin less than 7.5 and 3 were hypotensive on admission (Blood Pressure less than 100/70 $\mathrm{mm} \mathrm{Hg}$ ). All these 10 patients improved and were discharged. There were 5 patients with severe malaria who were documented to have been advised exchange transfusion and refused and 1 patient who received one unit of exchange transfusion and refused further transfusion. Of these 1 patient died and 4 recovered. Two patients $(1$ child) received 
blood transfusion in addition to the exchange transfusion. 18 patients received blood transfusion instead of exchange transfusion as the haemoglobin was low or the patient was not stable enough for an exchange.

There were 108 patients who were categorised into the severe malaria group of which 42 were above the age of 18 years and 66 were below. $76.2 \%$ of the adult patients were males, whereas there was no difference between the males and females in children. The average age of the adults with severe malaria was 31.84 years. 44 patients (22 adults and 22 children) received exchange transfusion. Parasitic index (PI) was available only for 59 patients. The average PI was 18.05 with 26 patients having a PI more than $10 \%$ $(44.07 \%)$.The measured characters of all the patients with severe malaria are in Table 1.

Table 1

\begin{tabular}{|l|c|c|c|}
\hline $\begin{array}{l}\text { Variable (number of valid } \\
\text { entries) }\end{array}$ & $\begin{array}{c}\text { Exchange transfusion } \\
(\mathrm{n}=22)\end{array}$ & $\begin{array}{c}\text { No exchange } \\
\text { transfusion }(\mathrm{n}=20) \\
\mathrm{N}(\% / \mathrm{Mean} \pm \mathrm{SD})\end{array}$ & $\begin{array}{c}\mathrm{P}- \\
\text { Value }\end{array}$ \\
\hline Systolic Blood Pressure & $102.07 \pm 15.89$ & $104.67 \pm 23$ & 0.81 \\
Diastolic Blood Pressure & $63.45 \pm 12.03$ & $66 \pm 13.54$ & 0.40 \\
Fever Duration (days) (42) & $4.65 \pm 2.21$ & $4.58 \pm 2.08$ & 0.86 \\
Jaundice (42) & $25(56.8)$ & $19(29.7)$ & 0.005 \\
Breathing difficulty (42) & $2(4.5)$ & $3(4.7)$ & 0.972 \\
Altered Sensorium (42) & $27(61.4)$ & $27(42.2)$ & 0.05 \\
Seizures (42) & $7(15.9)$ & $11(17.2)$ & 0.86 \\
Bleeding manifestations(42) & $5(11.4)$ & $12(18.8)$ & 0.3 \\
PI/hpf (23) & $31.31 \pm 24.87$ & $23 \pm 21.13$ & 0.22 \\
Parasite-pure falciparum & $26(59.1)$ & $45(70.3)$ & 0.23 \\
Mixed infection & $18(40.9)$ & $19(29.7)$ & 0.23 \\
Hemoglobin (gm\%) (64) & $8.93 \pm 2.64$ & $9.41 \pm 3.12$ & 0.355 \\
Platelet (cumm) (57) & $77707 \pm 46865$ & $102789 \pm 71979$ & 0.087 \\
Blood sugar (rbs mg\%) (62) & $115.93 \pm 40.14$ & $116.35 \pm 52.66$ & 0.87 \\
Potassium (58) & $4.24 \pm 0.85$ & $3.95 \pm 0.65$ & 0.10 \\
Creatinine (mg\%) (57) & $1.86 \pm 1.19$ & $1.12 \pm 0.56$ & 0.001 \\
Total bilirubin (mg\%) (23) & $10.16 \pm 9.55$ & $4.06 \pm 4.38$ & 0.034 \\
SGPT (pt) (23) & $57.23 \pm 33.21$ & $60.92 \pm 64.06$ & 0.45 \\
SGOT (ot) (23) & $112.34 \pm 74.49$ & $94.7 \pm 70.97$ & .044 \\
Decreased urine output (41) & $15(34.9)$ & $6(9.7)$ & 0.001 \\
Blood transfusion (42) & $2(4.5)$ & $21(32.8)$ & 0.000 \\
Hospital days (42) & $7.39 \pm 5.73$ & $6.19 \pm 3.32$ & 0.37 \\
Deteriorated (12) & $16(36.4)$ & $13(20.3)$ & 0.064 \\
\hline
\end{tabular}

The patients in the groups who received exchange transfusion were found to have higher incidence of jaundice (p), altered sensorium and decreased urine output. They also had higher serum creatinine, bilirubin and SGOT. More patients with severe malaria who received exchange transfusion deteriorated than those who did not which may reflect the more severe patients who got exchange transfusion.

The data of all patients with falciparum malaria was also analysed to identify if any of the variables that were looked at could have a prognostic value. This analysis is depicted in Table 2. 
Table 2. Predictors of adverse outcome

\begin{tabular}{|c|c|c|c|}
\hline Variable & $\begin{array}{c}\text { Deteriorated }(\%) \\
(\mathrm{n}=40)\end{array}$ & $\begin{array}{c}\text { Recovered }(\%) \\
(\mathrm{n}=179)\end{array}$ & P-Value \\
\hline Fever Duration (days) & $5.26 \pm 2.61$ & $4.61 \pm 2.37$ & \\
\hline Jaundice & $18(45)$ & $39(21.8)$ & 0.005 \\
\hline Breathing difficulty & $2(5)$ & $10(5.6)$ & 1.000 \\
\hline Altered Sensorium & $20(50)$ & $34(19.1)$ & 0.000 \\
\hline Seizures & $5(12.5)$ & $13(7.3)$ & 0.335 \\
\hline Bleeding manifestations & $2(5)$ & $15(8.4)$ & 0.745 \\
\hline BP Systolic & $110 \pm 16.49$ & $104.79 \pm 18.69$ & 0.154 \\
\hline BP Diastolic & $69.62 \pm 9.99$ & $67.01 \pm 12.51$ & 0.248 \\
\hline Hemoglobin (gm\%) & $8.66 \pm 3.14$ & $10.30 \pm 2.589$ & 0.000 \\
\hline Platelet (cumm) & $96176 \pm 59901$ & $106864 \pm 58304$ & 0.253 \\
\hline Blood sugar (rbs) (mg\%) & $107.11 \pm 51.63$ & $119.73 \pm 48.88$ & 0.091 \\
\hline Creatinine $(\mathrm{mg} \%)$ & $1.53 \pm 1.02$ & $1.32 \pm 0.73$ & 0.563 \\
\hline Serum bilirubin (tb) $(\mathrm{mg} \%)$ & $9.68 \pm 9.46$ & $4.47 \pm 6.66$ & 0.015 \\
\hline SGOT (ot) & $125.99 \pm 78.88$ & $78.61 \pm 73.66$ & 0.011 \\
\hline $\operatorname{SGPT}(\mathrm{pt})$ & $50.28 \pm 29.10$ & $53.36 \pm 50.43$ & 0.491 \\
\hline Decreased urine output & $15(38.5)$ & $6(3.5)$ & 0.000 \\
\hline Chest X-ray abnormality & $3(15.79)$ & $14(13.46)$ & 0.726 \\
\hline Blood transfusion & $5(12.5)$ & $25(14.0)$ & 1.000 \\
\hline Hospital days & $3.15 \pm 1.89$ & $6.74 \pm 3.60$ & 0.000 \\
\hline Exchange Transfusion & $16(40)$ & $38(21.2)$ & 0.024 \\
\hline
\end{tabular}

\section{Discussion}

The patients in both groups were similar with regard to their age, sex, the duration of fever and the blood pressure. Nearly $2 / 3^{\text {rds }}$ of the adult patients were males. This could be due to adult males spending more time outside, especially in the forests. The rate of mixed infection withboth Plasmodium falciparum and Plasmodium vivax has been reported to be $5-10 \% .{ }^{19}$ The high incidence $(33.79 \%)$ of mixed infection in our study is remarkable. This could be due to a high incidence of asymptomatic infection in the community as reported in a study conducted in the Amazon basin..$^{20}$ Malaria control in the region is still in the fledgling stage and community based measures like mosquito nets and DDT spraying are not widespread and a community based study may provide valuable information of this problem. Concomitant infection with $P$. vivax has been reported to lessen the severity of $P$. falciparum infection. ${ }^{21}$ However, this was not borne out in our study where there was similar incidence of mixed infection in both groups $(34.26 \%$ in the severe group and $33.33 \%$ in the non-severe group).

The mean duration of fever prior to admission was 4.72 days. This gives an insight into the health seeking behaviour in the area and a possible explanation for the high morbidity and mortality associated with malaria infection. The average age of the adults with malaria infection was 31.84 years indicating the burden the disease places on the young and productive members of the community.

The different variables were cross-tabulated with the outcome to identify if there were any prognostic indicators for a patient with malaria. The expected indices like jaundice, anaemia and renal failure were statistically significant in predicting an adverse outcome. We also found that a raised SGOT significantly increased the risk of an adverse outcome.

Of the adults with severe malaria, 3 patients out of $20(15 \%)$ who did not receive exchange transfusion deteriorated compared to 9 patients out of 22 (40.9\%) who did receive exchange transfusion. 10 children out of $44(22.7 \%)$ who did not receive exchange transfusion and 7 out of $22(31.8 \%)$ who 
did receive exchange transfusion deteriorated. Overall, 16 patients (36.4\%) who received exchange transfusion and $13(20.3 \%)$ of patients who did not deteriorated. This study did not demonstrate any survival benefit for patients who were treated with exchange transfusion as an adjunct to anti-malarials. In fact, paradoxically, a higher percentage of patients who received exchange transfusion had an adverse outcome.

However, an inference that exchange transfusion has no role in the treatment of malaria cannot be drawn for a number of reasons. Firstly, the patient selection for exchange transfusion was not randomised and there were no objective criteria for choosing to transfuse. Patients were advised exchange transfusion based on a subjective assessment of the severity of their disease and if there were no donors or the patient or relatives refused transfusion for any reason, the procedure was withheld. There were only 10 patients who received exchange transfusion who did not fit the criteria for severe malaria, which indicates that the assessment of the severity of illness by the medical team was by and large accurate.

The 2 groups of adult patients (who did and did not receive exchange transfusion) showed some dissimilarities on analysis with regard to jaundice, neck stiffness, sensorium, urine output, serum creatinine, bilirubin and SGOT. The patients who received exchange transfusion thus appear to be more 'sick' in terms of the parameters measured although these differences did not always reach statistical significance. This could explain the higher rate of adverse outcomes among the patients who received exchange transfusion.

Another possible reason for the apparent lack of survival benefit with exchange transfusion was that the volume of blood exchanged was only 350 $\mathrm{ml}$ in most cases. 7 patients received $700 \mathrm{ml}$ and 3 patients received $1050 \mathrm{ml}$. The volume of blood to be exchanged during the transfusion has not been standardised and different authors use different volumes. Recent data showing good response to exchange transfusion comes from centres where high volume is exchanged (up to 5 pints of packed cells and 3 units of Fresh Frozen Plasma) ${ }^{22,23 .}$
This volume of blood to be exchanged required high quality intensive care as well as large amounts of blood products which are not always available in secondary level hospitals of developing countries where a majority of patients with severe malaria are treated. A randomised, controlled trial in this setting is urgently required to clarify the role, if any, of low volume exchange transfusion. This will be a valuable tool if it proves to be useful on treating severe malaria and if it does not, will save large amounts of precious blood products that can be used for other more deserving illnesses.

\section{Conclusion}

This study did not show a significant difference in the outcomes of patients who did or did not receive exchange transfusion. However the patients who received exchange transfusion were more severely ill based on the indices measured. The strong prognostic indicators for poor outcome were severe jaundice and transaminitis, decreased urine output, altered sensorium and anaemia (Haemoglobin less than 8)

\section{Reference}

1. Malaria Facts. Centers for Disease Control and Prevention.

2. Ashwani Kumar, Neena Valecha, Tanu Jain, Aditya P. Dash. Burden of Malaria in India: Retrospective and Prospective View. Am. J. Trop. Med. Hyg., 77 (6_Suppl), 2007,pp. 69-78

3. Dondorp AM, Day NP. The treatment of severe malaria.Trans R Soc Trop Med Hyg. 2007;101:633-4

4. White NJ. The treatment of malaria. NEJM. 12.9.1996;335:11:800-806

5. Arjen M. Dondorp et al, M.D.Artemisinin Resistance in Plasmodium falciparum Malaria. Volume 361:455-467 July 30, 2009 Number 5

6. Zucker, JR, Campbell, CC. Malaria. Principles of prevention and treatment. Infect Dis Clin North Am 1993; 7:547. 
7. G.D. Burchard et al. Exchange blood transfusion in severe facliparum malaria: Retrospective analaysis of 61 patients treated with compared to 63 patients treated without exchange transfusion. Trop. Med and Int. Health, Vol 2 No 8 PP 733-740, Aug 97

8. WhiteNJ. What is the future of exchange transfusion in severe malaria? J Infect 1999;39:185-6.

9. Dondorp AM, Angus BJ, Hardeman MR, Chotivanich KT, Silamut K, Ruangveerayuth R, Kager PA, WhiteNJ, Vreeken J. Prognostic significance of reduced red blood cell deformability in severe falciparum malaria. Am J Trop Med Hyg 1997;57:507-11.

10. Kumar S, Karnad DR, Vaingankar J, Thatte UM, Krishnan A, Rege NN. Serum tumour necrosis factor alpha levels in severe malaria: effect of partial exchange transfusion. Intensive Care Med 2003;29:1857-8.

11. World Health Organization Severe falciparum malaria. Trans $R$ Soc Trop Med Hyg.2000;94(suppl 1):S1-S90.

12. Hoontrakoon S, Suputtamongkol Y. Exchange transfusion as an adjunct to the treatment of severe falciparum malaria. Trop Med Int Health 1998;3:156-61.

13. Nieuwenhuis JA, Meertens JH, Zijlstra JG, Ligtenberg JJ, Tulleken JE, van der Werf TS. Automated erythrocytapher-esis in severe falciparum malaria: a critical reappraisal.Acta Trop 2006;98:201-6.

14. Andrej Trampuz, Matjaz Jereb, Igor Muzlovic, and Rajesh M Prabhu.Clinical review: Severe malaria. Crit Care. 2003; 7(4): 315-323.

15. Riddle MS, Jackson JL, Sanders JW, Blazes DL. Exchange transfusion as an adjunct therapy in severe Plasmodium falciparum malaria: a meta-analysis. Clin Infect Dis. 2002;34:1192-1198. doi: $10.1086 / 339810$.
16. WHO. Guidelines for the treatment of malaria, second edition, 2010

17. Chloroquine resistant P. falciparum malaria in Assam and Meghalaya. Barkakaty $\underline{B N}, \underline{\text { Kalita PC}}, \underline{\text { Das S}}, \underline{\text { Talukdar AC. }}$

18. Guidelines for Diagnosis and Treatment of malaria in India, 2009. Govt. of India Publication

19. Blaise Genton, Valérie D' Acremont, Lawrence Rare, Kay Bae, John C. Reeder, Michael P. Alpers, Ivo Müller. Plasmodium vivax and Mixed Infections Are Associated with Severe Malaria in Children: A Prospective Cohort Study from Papua New Guinea. PLoS Medicine. June 2008

20. Fabiana P. Alves, Rui R. Durlacher, Maria J. Menezes, Henrique Krieger, Luiz $\mathrm{H}$. Pereira da Silva, Erney P. Camargo. High prevalence of Aymptomatic Plasmodium vivax and Plasmodium falciparum infections in native Amazonian populations. Am. J. Trop. Med. Hyg., 66(6), 2002, pp. 641-648

21. Luxemburger C, Ricci F, Raimond D, Bathet S, White NJ. The epidemiology of severe malaria in an area of low transmission in Thailand. Trans R. Soc Trop Med Hyg. 1997;91:256-62.

22. Perry J.J. van Genderen, Dennis A. Hesselink, Jacob M. Bezemer, Pieter J. Wismans and David Overbosch. Efficacy and safety of exchange transfusion as an adjunct therapy for severe Plasmodium falciparum malaria in nonimmune travelers: a 10-year single-center experience with a standardized treatment protocol. TRANSFUSION 2010;50:787-794.

23. Suresh G. Shelat, Jason P Lott, and Matthew S. Braga. Considerations on the use of adjunct red blood cell exchange transfusion in the treatment of severe Plasmodium falciparum malaria. TRANSFUSION 2010;50:875-880. 